\title{
Caractérisation Des Paramètres Agroclimatiques Clés De La Saison Culturale En Zone De Contact Forêt- Savane De Côte-d'Ivoire
}

\author{
Brou Kouamé \\ Centre National de Recherche Agronomique (CNRA), \\ Laboratoire Central Sols, Eaux et Plantes, Programme Gestion \\ Durable des Sols et Maîtrise de l'Eau
}

Jean-Noël Ehounou

Université Félix Houphouët-Boigny, Laboratoire de Sciences et Techniques de l'Eau et de l'Environnement (LSTEE), Côte d'Ivoire

\section{Koffi Emmanuel Kassin}

Centre National de Recherche Agronomique (CNRA), Programme Cacao

\section{Charles Sépka Dekoula}

Université Félix Houphouët-Boigny, Laboratoire de Sciences et Techniques de l'Eau et de l'Environnement (LSTEE), Côte d'Ivoire

\section{Guy Fernand Yao \\ Emmanuel Kouadio N'goran \\ Brou Julien Kouakou \\ Boaké Koné}

Centre National de Recherche Agronomique (CNRA),

Laboratoire Central Sols, Eaux et Plantes,

Programme Gestion Durable des Sols et Maîtrise de l'Eau, Programme

coton, Côte d'Ivoire, Programme Cacao

\section{Nagnin Soro}

Université Félix Houphouët-Boigny, Laboratoire de Sciences et Techniques de l'Eau et de l'Environnement (LSTEE), Côte d'Ivoire

\begin{abstract}
In the Central region of Côte d'Ivoire, contact area between the South forest estate and savanna's area in the North, the climate is a major concern for people. Since the late 1960s, the constant changes in rainfall amounts make it difficult for the various agricultural operations in the area. Before the natural instability of the rainfall regime and the extreme variability of agro-climatic parameters, farmers are no longer able to detect the probable dates of start and end of the rainy seasons that are essential to optimize agricultural production.
\end{abstract}


From the descriptive statistical analysis of rainfall data from the stations of Bouaké, Béoumi, Katiola and Dabakala over the period 1961-2000, this study focuses on assessing the dynamics of agro-climatic key factors of the growing season in this transition zone. The results show an earlier start and a later end of the rainy season in Bouaké and Béoumi. On the contrary, the seasons started later and ended earlier in the localities of Katiola and Dabakala, and the dry sequences, during the rainy seasons, are longer in these localities.

Keywords: Key agro-climatic factors, cropping season, Center of Côte d'Ivoire

\section{Résumé}

Dans la région Centre de la Côte d'Ivoire, zone de contact entre le domaine forestier du Sud et le domaine savanicole du Nord, le climat constitue un sujet de préoccupation majeur pour les populations. Depuis la fin des années 1960, les variations constantes des hauteurs pluviométriques rendent difficiles les différentes opérations agricoles dans la zone. Face à l'instabilité naturelle du régime pluviométrique et l'extrême variabilité des paramètres agroclimatiques, les paysans n'arrivent plus à déceler les dates probables de début et de fin des saisons pluvieuses qui sont pourtant essentielles pour optimiser les productions agricoles. À partir de l'analyse statistique descriptive des données pluviométriques issues des stations à Bouaké, Béoumi, Katiola et Dabakala, sur la période 1961-2000, cette étude évalue la dynamique des facteurs agroclimatiques clés de la campagne agricole dans cette zone de transition. Les résultats révèlent un début plus précoce et une fin plus tardive des saisons pluvieuses à Bouaké et à Béoumi. Les saisons pluvieuses sont donc plus longues dans le Sud de la région. De même, les cumuls pluviométriques et les jours pluvieux au cours des saisons y sont plus importants. Au contraire, les saisons débutent plus tardivement et s'achèvent plus précocement dans les localités de Katiola et de Dabakala et les séquences sèches, au cours des saisons pluvieuses, sont plus longues dans ces localités.

Mots-clés: Facteurs agroclimatiques clés, saison culturale, Centre de la Côte d'Ivoire

\section{Introduction}

Les changements climatiques ont entraîné de nombreux bouleversements autour du monde. En effet, il ne se passe plus une année sans que l'on observe l'un des effets néfastes de ces nouvelles conditions climatiques sur la planète (pluies diluviennes, sécheresses extrêmes, ouragans, Tsunamis). À l'échelle du globe, l'augmentation des concentrations des gaz à effet de serre, principale cause du réchauffement climatique, affecte la 
biodiversité, le niveau des mers, la dynamique des glaciers et des océans (Tourquebiau, 2015). En Afrique, et notamment en Côte d'Ivoire, le dérèglement climatique se manifeste surtout par des sécheresses récurrentes et une perturbation de la saisonnalité et du régime des précipitations. Cette instabilité pluviométrique est accompagnée par des déficits pluviométriques de 1'ordre de $20 \%$ à $30 \%$ et des baisses des débits des cours d'eau (Servat et al., 1997 ; Paturel et al., 1997 ; Brou et al., 1998 ; Soro et al., 2014). La baisse pluviométrique, amorcée dans les pays du golfe de Guinée dès la fin des années 1960, s'est intensifiée en Côte d'Ivoire au cours des années 1980 et 1990, avant de connaître une certaine rémission durant les années 2000 (Noufé, 2015).

À l'instar de l'ensemble du pays, la région Centre de la Côte d'Ivoire subit les aléas du climat et la récession pluviométrique induit une variabilité des dates de début, de fin et de durée des saisons (Diomandé, 2013). Dans cette zone où l'agriculture demeure la principale activité génératrice de revenus, les incertitudes climatiques constituent une menace certaine pour les communautés locales qui les perçoivent à juste titre comme des facteurs potentiels de réduction des rendements et des ressources financières des ménages.

La présente étude s'est donc assignée pour objectif de caractériser les paramètres agroclimatiques dont dépend la réussite de la campagne agricole afin d'accroître la résilience des paysanneries du Centre dans le contexte actuel des changements climatiques.

\section{Matériels et méthodes \\ Site d'étude}

Située dans le Centre de la Côte d'Ivoire, entre les longitudes $3^{\circ} 54^{\prime}$ et $5^{\circ} 42^{\prime} \mathrm{W}$ et les latitudes $7^{\circ} 18^{\prime}$ et $9^{\circ} 24^{\prime} \mathrm{N}$, la zone d'étude s'étend sur une superficie de $25914 \mathrm{~km}^{2}$. L'étude concerne en particulier les localités de Bouaké, Béoumi, Katiola et Dabakala (Figure 1). C'est une zone intermédiaire entre les plateaux du Nord et les plaines du Sud d'une part et entre les forêts humides à courtes saisons sèches et les savanes à longue saison sèche, d'autre part (Kanga, 2017). Le climat y est de type «soudano-guinéen » (Dolidon, 2007). Les températures oscillent autour de $27{ }^{\circ} \mathrm{C}$ avec des écarts de l'ordre de 3 à $5^{\circ} \mathrm{C}$. L'évaporation décadaire moyenne varie entre 35 et $55 \mathrm{~mm}$ en saison de pluie et l'humidité relative fluctue entre 70 et $80 \%$. Le régime pluviométrique est de type bimodal avec des précipitations très irrégulières qui atteignent un total annuel moyen de l'ordre de 1100 mm (Kouamé, 1992). La végétation prédominante est la savane dite guinéenne (étendue de hautes herbes d'environ 1,5 m comprises entre les forêts denses et les forêts claires parsemées d'arbres). 


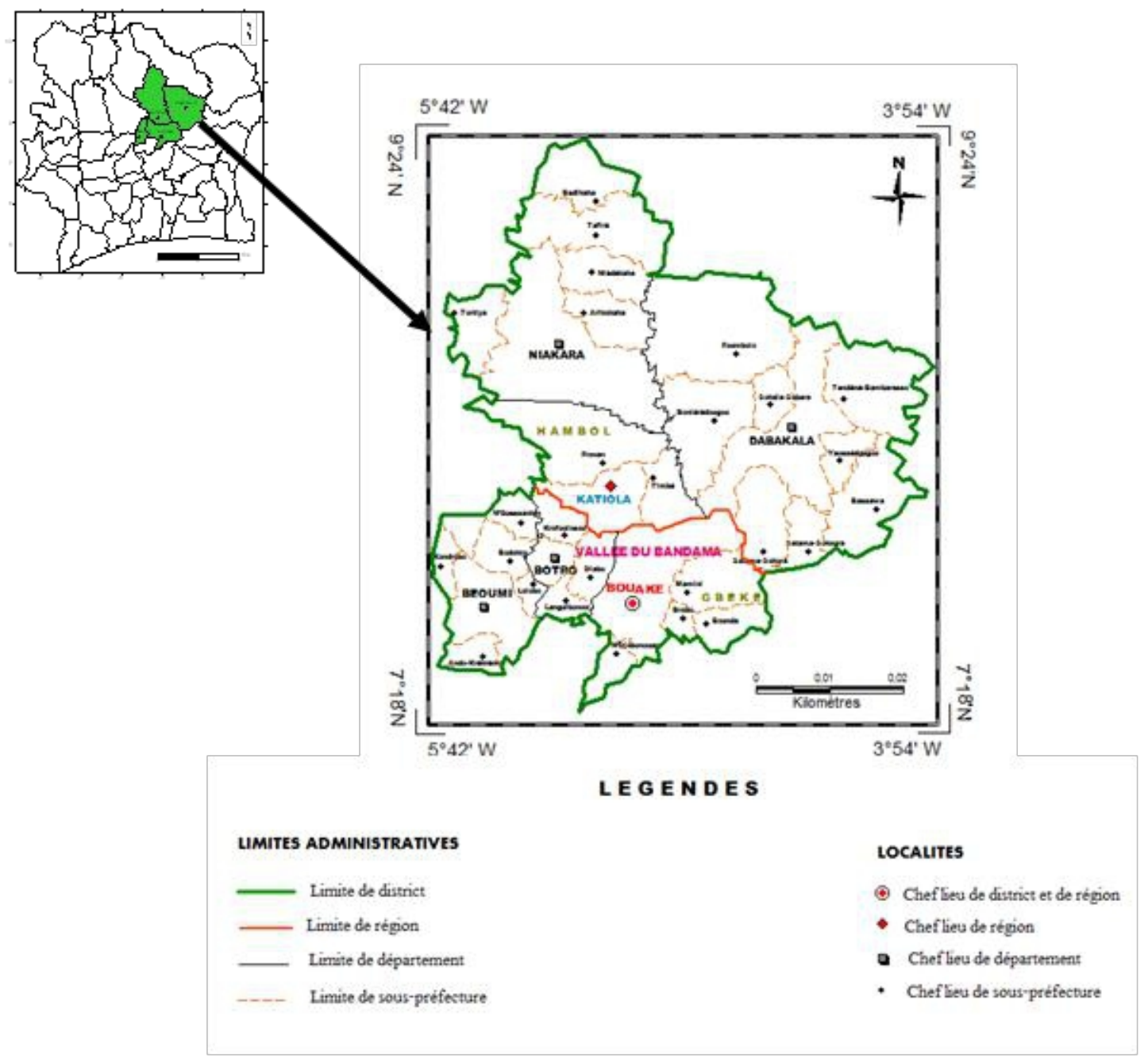

Figure 1. Localisation de la zone d'étude

\section{Données}

Les données pluviométriques sont issues de la Société d'Exploitation et de Développement Aéroportuaire, Aéronautique et Météorologique (SODEXAM) et du Service Météorologique du Centre National de Recherche Agronomique (CNRA). Afin de travailler sur des séries relatives à des périodes d'observations identiques, il a été retenu une période temporelle commune sur quatre (4) stations qui s'étend de 1961 à 2000. Toutefois, il convient de noter qu'à l'exception de la Station de Recherche sur les Cultures Vivrières du CNRA à Bouaké, les autres stations ont présenté des données journalières manquantes dans des proportions relativement faibles (n'excédant pas 3\% de la base de données dans chacune des localités concernées (Béoumi, Katiola et Dabakala). 
En l'absence de méthodes d'estimation fiables à $100 \%$, les années comportant un nombre trop élevé de données manquantes ont été supprimées de la base de données. En effet, la série considérée pour chaque station (19612000) étant suffisamment longue, cette opération n'a pas eu de répercussion notable sur la fiabilité de nos résultats. En outre, il faut souligner que les graves évènements socio-politiques survenus en Côte d'Ivoire entre 1999 et 2010 ont rendu inopérantes les collectes de données sur les différents postes de mesure de la zone d'étude jusqu'en 2011. Le choix de la période 1961-2000 s'est donc imposé en raison du manque de données pluviométriques récentes pour étudier la période actuelle (2001 à 2017).

\section{Méthodes}

La détermination des paramètres agroclimatiques clés de la saison agricole a été faite à l'aide du logiciel Instat+ v3.030, à partir de l'analyse statistique fréquentielle. Des critères établis sur la base des informations recueillies sur les pratiques paysannes dans la région ont permis de déterminer les paramètres fondamentaux dont dépend le succès d'une campagne agricole. Il s'agit, entre autres :

- des dates de début et de fin des saisons de pluies;

- de la longueur des saisons de pluies et du cumul pluviométrique saisonnier;

- du nombre de jours de pluie saisonniers et des séquences sèches maximales au cours des saisons pluvieuses.

\section{$>$ Dates de début et de fin des saisons pluvieuses}

La grande saison des pluies débute à partir du $1^{\text {er }}$ février, lorsque la quantité de pluie recueillie en trois jours consécutifs est au moins égale à $20 \mathrm{~mm}$, et lorsqu'aucune période de sécheresse de plus de sept jours n'intervient dans les 30 jours suivants. La petite saison des pluies démarre, après le $1^{\text {er }}$ août, lorsque la quantité d'eau recueillie est au moins égale à $20 \mathrm{~mm}$, sans aucune période de sécheresse de plus de sept jours dans les 30 jours suivants.

La fin de la grande saison pluvieuse a lieu lorsque, après le $1^{\text {er }}$ juillet, l'on n'enregistre aucune pluie pendant au moins 20 jours consécutifs. La petite saison des pluies s'achève à partir du $1^{\text {er }}$ novembre, lorsqu'il n'y a plus de pluie pendant au moins 20 jours consécutifs.

\section{Longueur des hivernages et cumul pluviométrique saisonnier}

La longueur des saisons des pluies (grande saison et petite saison) résulte de la différence entre les fins et les débuts de ces saisons.

Le cumul pluviométrique saisonnier est la quantité totale de pluie recueillie au cours d'une saison pluvieuse. 


\section{$>$ Nombre de jours de pluie saisonniers et séquences sèches maximales}

L'analyse des nombres de jours de pluie saisonniers et décadaires a été réalisée conjointement à l'aide du logiciel Instat+ version 3.030 et Excel version 2013 pour Windows. Selon la norme établie par l'Organisation Météorologique Mondiale (OMM), un jour est considéré comme pluvieux lorsque celui-ci présente une hauteur de pluie d'au moins $1 \mathrm{~mm}$. Les séquences sèches les plus longues pendant les deux saisons des pluies ont été générées à l'aide du logiciel Instat+v3.030. Une séquence sèche est une succession de jours consécutifs durant lesquels les pluies sont inférieures à un seuil de $1 \mathrm{~mm}$.

\section{Résultats}

\section{Dates de démarrage des saisons pluvieuses}

À l'exception de Bouaké (où le démarrage de la saison a lieu le 24 mars), la grande saison des pluies démarre en moyenne dans la première décade du mois d'avril, sur l'ensemble des stations étudiées. Son démarrage est plus précoce à Béoumi ( $1^{\mathrm{er}}$ avril) et devient plus tardif au fur et à mesure que l'on remonte vers le Nord en direction des localités de Katiola ( 2 avril) et de Dabakala (10 avril). À Bouaké, la date de début de la grande saison des pluies se situe entre le 11 mars et le 10 avril dans $80 \%$ des années. À Dabakala où la grande saison des pluies est la plus tardive, le début de la saison se situe, quatre années sur cinq, entre le 24 mars et le 27 avril (Tableau I). À Béoumi, le début de la grande saison des pluies se situe, quatre années sur cinq, entre le 14 mars et le 16 avril. Quant à Katiola, le démarrage de la saison a lieu entre le 18 mars et le 19 avril dans les mêmes proportions. En général, sur l'ensemble des quatre stations étudiées, le démarrage de la grande saison des pluies se situe entre la deuxième décade de mars et la troisième décade d'avril, quatre années sur cinq (Tableau I).

La petite saison des pluies démarre en moyenne entre la première et la dernière décade d'août sur l'ensemble des quatre stations. Il a été observé un démarrage précoce de la petite saison des pluies à Bouaké et à Béoumi et un début tardif en remontant vers le Nord à Katiola et à Dabakala. À Bouaké où la saison commence plus tôt, la date de début de la petite saison des pluies est comprise, quatre années sur cinq, entre le $1^{\mathrm{er}}$ août et le 13 août. À Dabakala, où elle est plus tardive, la date de démarrage de la petite saison des pluies se situe entre le 05 août et le 04 septembre. D'une manière générale, le début de la petite saison des pluies dans la zone intervient, quatre années sur cinq, entre la deuxième décade d'août et la première décade de septembre (Tableau I). 
Tableau I. Moyennes et percentiles des dates de début des saisons des pluies sur les 4 stations au cours de la période 1961-2000

\begin{tabular}{|c|c|c|c|c|}
\hline Station & BOUAKE & BEOUMI & KATIOLA & DABAKALA \\
\hline \multicolumn{5}{|c|}{ GRANDE SAISON } \\
\hline Moyenne & 24 mars & $1^{\mathrm{er}}$ avril & 02 avril & 0 avril \\
\hline Écart type & 15 & 23 & 21 & 24 \\
\hline Coefficient de variation (\%) & 18 & 25 & 22 & 23 \\
\hline Percentile $20 \%$ & 11 mars & 14 mars & 18 mars & 24 mars \\
\hline Percentile $50 \%$ & 21 mars & 24 mars & 31 mars & 07 avril \\
\hline Percentile $80 \%$ & 10 avril & 16 avril & 19 avril & 27 avril \\
\hline \multicolumn{5}{|c|}{ PETITE SAISON } \\
\hline Moyenne & 06 août & 17 août & 22 août & 20 août \\
\hline Écart type & 11 & 10 & 9 & 14 \\
\hline Coefficient de variation $(\%)$ & 5 & 4 & 4 & 6 \\
\hline Percentile $20 \%$ & $1^{\mathrm{er}}$ août & 10 août & 14 août & 05 août \\
\hline Percentile $50 \%$ & 07 août & 18 août & 18 août & 17 août \\
\hline Percentile $80 \%$ & 13 août & 26 août & $1^{\mathrm{er}}$ septembre & 04 septembre \\
\hline
\end{tabular}

\section{Dates de fin des saisons pluvieuses}

La fin de la grande saison pluvieuse intervient en moyenne entre le 06 juillet et le 11 juillet sur l'ensemble des quatre stations. Elle est plus précoce à Bouaké et à Dabakala et plus tardive à Béoumi et Katiola. Dans la station de Dabakala où elle arrive plus tôt, la fin de la grande saison pluvieuse se situe entre le 19 juin et le 20 juillet, quatre années sur cinq. À Béoumi, où elle est plus tardive, la fin de la grande saison des pluies varie entre le 24 juin et le 02 août dans les mêmes proportions. Plus généralement, sur l'ensemble des quatre stations, la fin de la grande saison pluvieuse intervient quatre années sur cinq entre le 19 juin (Dabakala) et le 02 août (Béoumi) (Tableau II). La petite saison des pluies finit en moyenne entre le 12 octobre et le 17 novembre sur l'ensemble des quatre stations. La fin de la petite saison intervient plus tôt à Bouaké et à Dabakala. À Bouaké où la petite saison des pluies se termine plus tôt, sa fin intervient, quatre années sur cinq, entre le 28 septembre et le 28 octobre. À Béoumi, où elle est plus tardive, la petite saison des pluies s'achève, quatre années sur cinq, entre le 18 octobre et le 17 novembre. D'une manière générale, pour l'ensemble des stations étudiées, la fin de la petite saison des pluies se situe, quatre années sur cinq, entre le 28 octobre (Bouaké) et le 17 novembre (Béoumi) (Tableau II).

Tableau II. Moyennes et percentiles des dates de fin des saisons des pluies sur les 4 stations au cours de la période 1961-2000

\begin{tabular}{lcccc}
\hline Station & BOUAKE & BEOUMI & KATIOLA & ABAKALA \\
\hline \multicolumn{4}{c}{ GRANDE SAISON } \\
\hline Moyenne & 07 juillet & 11 juillet & 08 juillet & 06 juillet \\
Écart type & 15 & 19 & 19 & 16 \\
Coefficient de variation $(\%)$ & 8 & 10 & 10 & 8
\end{tabular}


Percentile $20 \%$

Percentile $50 \%$

Percentile $80 \%$

$$
\begin{array}{cc}
24 \text { juin } & 24 \text { juin } \\
03 \text { juillet } & 07 \text { juillet } \\
22 \text { juillet } & 02 \text { août }
\end{array}
$$

20 juin

06 juillet

27 juillet
19 juin

06 juillet

20 juillet

Moyenne
Écart type
Coefficient de variation (\%)
Percentile $20 \%$
Percentile $50 \%$
Percentile $80 \%$

\section{PETITE SAISON}

\section{2 octobre}

19

7

28 septembre

14 octobre

28 octobre
02 novembre

15

5

18 octobre

03 novembre

17 novembre
17 octobre

17

6

04 octobre

18 octobre

30 octobre
21 octobre

14

5

09 octobre

18 octobre

04 novembre

\section{Longueur des saisons pluvieuses}

D'une manière générale, la grande saison des pluies est plus longue dans le Sud du domaine d'étude (Bouaké et Béoumi) que dans le Nord de la zone (Katiola et Dabakala). La longueur moyenne de la grande saison des pluies varie entre 87 et 106 jours sur l'ensemble des quatre stations. À Dabakala, où la moyenne est la plus faible, la longueur de la grande saison des pluies oscille entre 72 et 100 jours. À Bouaké, où elle est plus élevée, la longueur de la grande saison des pluies varie, quatre années sur cinq, entre 81 et 132 jours (Tableau III). À l'instar de la grande saison des pluies, les petites saisons des pluies sont plus longues à Béoumi et à Bouaké (Sud de la zone d'étude) et plus courtes dans le Nord de cette zone (Katiola et Dabakala). En moyenne, la longueur de la petite saison varie entre 56 et 76 jours. À Katiola, où elle est

\begin{tabular}{|c|c|c|c|c|}
\hline Station & BOUAKE & BEOUMI & KATIOLA & DABAKALA \\
\hline \multicolumn{5}{|c|}{ GRANDE SAISON } \\
\hline Moyenne & 106 jours & 101 jours & 97 jours & 87 jours \\
\hline Écart type & 25 & 26 & 27 & 19 \\
\hline Coefficient de variation $(\%)$ & 24 & 25 & 28 & 22 \\
\hline Percentile $20 \%$ & 81 jours & 78 jours & 74 jours & 72 jours \\
\hline Percentile $50 \%$ & 108 jours & 96 jours & 98 jours & 88 jours \\
\hline Percentile $80 \%$ & 132 jours & 127 jours & 122 jours & 100 jours \\
\hline \multicolumn{5}{|c|}{ PETITE SAISON } \\
\hline Moyenne & 66 jours & 76 jours & 56 jours & 62 jours \\
\hline Écart type & 17 & 15 & 17 & 18 \\
\hline Coefficient de variation $(\%)$ & 25 & 26 & 31 & 29 \\
\hline Percentile $20 \%$ & 51 jours & 65 jours & 44 jours & 43 jours \\
\hline Percentile $50 \%$ & 64 jours & 76 jours & 57 jours & 64 jours \\
\hline Percentile $80 \%$ & 81 jours & 91 jours & 72 jours & 80 jours \\
\hline
\end{tabular}
plus faible, la durée de la petite saison se situe, quatre années sur cinq, entre 44 et 72 jours. À Béoumi, où elle est plus élevée, quatre années sur cinq, la durée de la petite saison des pluies varie entre 65 et 91 jours (Tableau III).

Tableau III. Moyennes et percentiles des longueurs des saisons des pluies sur les 4 stations au cours de la période 1961-2000 


\section{Cumuls pluviométriques saisonniers}

Le cumul de la grande saison des pluies décroit de la partie Sud de la zone d'étude vers la partie Nord. La pluviométrie saisonnière moyenne varie entre 393 et 499 mm sur l'ensemble des quatre stations. À Dabakala, où il est enregistré la plus faible moyenne, le cumul de la grande saison des pluies atteint ou dépasse $287 \mathrm{~mm}$, quatre années sur cinq, et n'excède pas $485 \mathrm{~mm}$, dans les mêmes proportions. Elle varie donc entre $287 \mathrm{~mm}$ et $485 \mathrm{~mm}$ quatre années sur cinq. À Bouaké, où la moyenne est la plus élevée, le cumul oscille entre 371 et $678 \mathrm{~mm}$, quatre années sur cinq (Tableau IV). Tout comme le cumul de la grande saison des pluies, celui de la petite saison est plus élevé dans le Sud du domaine d'étude (Bouaké et Béoumi) et a tendance à décroître lorsque l'on va vers le Nord (Katiola et Dabakala). La moyenne varie entre 333 et $401 \mathrm{~mm}$ sur l'ensemble des quatre stations. À Katiola, où la plus faible moyenne est enregistrée, le cumul de la petite saison des pluies atteint ou dépasse $186 \mathrm{~mm}$ quatre années sur cinq. Le cumul est également inférieur à $468 \mathrm{~mm}$ dans $80 \%$ des cas, ce qui signifie que le cumul varie entre 186 et $468 \mathrm{~mm}$ quatre années sur cinq (Tableau IV).

Tableau IV. Moyennes et percentiles des cumuls pluviométriques (en $\mathrm{mm}$ ) des saisons des pluies sur les 4 stations au cours de la période 1961-2000

\begin{tabular}{|c|c|c|c|c|}
\hline Station & BOUAKE & BEOUMI & KATIOLA & DABAKALA \\
\hline \multicolumn{5}{|c|}{ GRANDE SAISON } \\
\hline Moyenne & 499 & 445 & 444 & 393 \\
\hline Écart type & 154 & 114 & 155 & 114 \\
\hline Coefficient de variation $(\%)$ & 31 & 26 & 35 & 29 \\
\hline Percentile $20 \%$ & 371 & 348 & 295 & 287 \\
\hline Percentile $50 \%$ & 469 & 430 & 402 & 413 \\
\hline Percentile $80 \%$ & 678 & 539 & 583 & 485 \\
\hline \multicolumn{5}{|c|}{ PETITE SAISON } \\
\hline Moyenne & 359 & 401 & 337 & 333 \\
\hline Écart type & 156 & 107 & 174 & 149 \\
\hline Coefficient de variation (\%) & 43 & 27 & 52 & 45 \\
\hline Percentile $20 \%$ & 194 & 314 & 186 & 205 \\
\hline Percentile $50 \%$ & 368 & 400 & 335 & 327 \\
\hline Percentile $80 \%$ & 501 & 478 & 468 & 474 \\
\hline
\end{tabular}

\section{Nombre de jours de pluie}

Le nombre de jours pluvieux pendant la grande saison des pluies est plus élevé dans le Sud de la zone d'étude et décroît à mesure que l'on va vers le Nord. La moyenne varie entre 20 et 29 jours. Les moyennes et les percentiles des nombres de jours pluvieux dans la grande saison pour les quatre stations sont consignés dans le Tableau V.

À Dabakala, où est enregistrée la plus faible moyenne, le nombre de jours de pluie atteint ou dépasse 16 jours, quatre années sur cinq, mais 
n'excède pas 25 jours dans les mêmes proportions. Il varie donc, quatre années sur cinq, entre 16 et 25 jours. Pendant la petite saison des pluies également le nombre de jours pluvieux est plus élevé dans le Sud du domaine d'étude (Bouaké et Béoumi) et plus faible lorsque l'on va vers les localités situées plus au Nord (Katiola et Dabakala). En moyenne, on dénombre 18 à 24 jours de pluie sur l'ensemble des quatre stations. À Dabakala, où la moyenne est la moins élevée, le nombre de jours pluvieux varie, dans $80 \%$ des années, entre 13 et 24 jours. À Bouaké, où la moyenne est la plus élevée, le nombre de jours pluvieux pendant la petite saison des pluies oscille entre 16 et 32 jours (Tableau V).

Tableau V. Moyennes et percentiles des nombres de jours pluvieux des deux saisons pluvieuses sur les 4 stations sur la période 1961-2000

\begin{tabular}{|c|c|c|c|c|}
\hline Station & BOUAKE & BEOUMI & KATIOLA & DABAKALA \\
\hline \multicolumn{5}{|c|}{ GRANDE SAISON } \\
\hline Moyenne & 29 jours & 25 jours & 24 jours & 20 jours \\
\hline Écart type & 8 & 7 & 8 & 6 \\
\hline Coefficient de variation (\%) & 29 & 27 & 36 & 30 \\
\hline Percentile $20 \%$ & 21 jours & 19 jours & 16 jours & 16 jours \\
\hline Percentile $50 \%$ & 29 jours & 26 jours & 23 jours & 21 jours \\
\hline Percentile $80 \%$ & 38 jours & 31 jours & 32 jours & 25 jours \\
\hline \multicolumn{5}{|c|}{ PETITE SAISON } \\
\hline Moyenne & 24 jours & 23 jours & 19 jours & 18 jours \\
\hline Écart type & 9 & 6 & 8 & 7 \\
\hline Coefficient de variation $(\%)$ & 36 & 27 & 45 & 39 \\
\hline Percentile $20 \%$ & 16 jours & 17 jours & 13 jours & 13 jours \\
\hline Percentile $50 \%$ & 24 jours & 22 jours & 18 jours & 18 jours \\
\hline Percentile $80 \%$ & 32 jours & 30 jours & 26 jours & 24 jours \\
\hline
\end{tabular}

\section{Séquences sèches maximales}

À l'inverse du nombre de jours de pluie, les deux localités septentrionales (Katiola et Dabakala) enregistrent les séquences sèches les plus longues pendant la grande saison pluvieuse. Les deux localités situées plus au Sud dans la zone d'étude (Bouaké et Béoumi), plus pluvieuses, présentent fort logiquement les séquences sèches les moins longues de la zone. La moyenne des séquences sèches les plus longues est de 10 jours pour Katiola et Dabakala et de 9 jours pour Bouaké et Béoumi. À Katiola et Dabakala, où la moyenne est plus élevée, le nombre de jours de la séquence sèche maximale oscille, dans $80 \%$ des cas, entre 6 et 12 jours (Tableau VI). Pendant la petite saison pluvieuse, les séquences sèches les plus longues se retrouvent également dans le Nord du domaine d'étude. La moyenne des séquences sèches maximales varie entre 9 et 11 jours sur l'ensemble des quatre stations. À Bouaké et Béoumi, où la moyenne est plus faible, la durée de la séquence 
sèche maximale se situe entre 5 et 13 jours, dans $80 \%$ des cas. À Katiola, où la moyenne est élevée, la longueur de la séquence sèche maximale oscille entre 5 et 19 jours, dans $80 \%$ des années (Tableau VI).

Tableau VI. Moyennes et percentiles des séquences sèches maximales pendant les deux saisons des pluies sur les 4 stations au cours de la période 1961-2000

\begin{tabular}{|c|c|c|c|c|}
\hline Station & BOUAKE & BEOUMI & KATIOLA & DABAKALA \\
\hline \multicolumn{5}{|c|}{ GRANDE SAISON } \\
\hline Moyenne & 9 jours & 9 jours & 10 jours & 10 jours \\
\hline Écart type & 4 & 4 & 5 & 4 \\
\hline Coefficient de variation $(\%)$ & 48 & 49 & 52 & 42 \\
\hline Percentile $20 \%$ & 6 jours & 6 jours & 6 jours & 6 jours \\
\hline Percentile $50 \%$ & 8 jours & 8 jours & 8 jours & 9 jours \\
\hline Percentile $80 \%$ & 11 jours & 12 jours & 12 jours & 12 jours \\
\hline \multicolumn{5}{|c|}{ PETITE SAISON } \\
\hline Moyenne & 9 jours & 9 jours & 11 jours & 12 jours \\
\hline Écart type & 5 & 5 & 9 & 8 \\
\hline Coefficient de variation $(\%)$ & 56 & 55 & 78 & 68 \\
\hline Percentile $20 \%$ & 5 jours & 5 jours & 5 jours & 5 jours \\
\hline Percentile $50 \%$ & 7 jours & 8 jours & 8 jours & 10 jours \\
\hline Percentile $80 \%$ & 13 jours & 13 jours & 19 jours & 16 jours \\
\hline
\end{tabular}

\section{Discussion}

À l'image des autres régions du pays, les activités agricoles dans le Centre de la Côte d'Ivoire sont essentiellement dépendantes du régime pluviométrique de la zone. Il va sans dire qu'une irrégularité des précipitations a des répercussions indéniables sur la production agricole.

La région Centre de la Côte d'Ivoire, constituée par la zone médiane communément appelée "V Baoulé », est une zone de transition entre le Sud forestier à deux saisons des pluies et les savanes du Nord caractérisées par une seule saison pluvieuse. Du fait de sa situation géographique, la région est donc naturellement influencée par le régime unimodal du Nord et le régime bimodal rencontré au Sud. La bande de territoire qui s'étend de part et d'autre de Bouaké, entre les $7^{\circ}$ et $9^{\circ}$ de latitude Nord, est donc une zone de contact qui, suivant les années, subira un régime à 2 ou 4 saisons et où, par suite, les prévisions concernant le climat annuel sont particulièrement difficiles (Diomandé, 2013). Cette instabilité du régime pluviométrique constitue un frein à la mise en place des différentes campagnes agricoles dans la région. Et pour cause, la date d'installation des pluies et la durée de la saison sont deux paramètres fondamentaux pour la planification des opérations agricoles, car ils déterminent, d'une part, la date de semis ou de mise en place des spéculations et, d'autre part, la durée de la période pendant laquelle les cultures peuvent bénéficier des précipitations, indépendamment des conditions d'alimentation hydrique de cette période. 
L'analyse des données pluviométriques journalières collectées sur les quatre stations concernées par la présente étude au cours de la période 19612000 a permis de mettre en évidence l'importante irrégularité pluviométrique dans la région, notamment au niveau des facteurs clés de la saison culturale. Le traitement des données pluviométriques au cours de cette période indique, en effet, un démarrage plus précoce des deux saisons pluvieuses dans les localités de Bouaké et de Béoumi et un début plus tardif au fur et à mesure que l'on gagne en latitude vers les localités septentrionales (Katiola et Dabakala). Cette instabilité pluviométrique a été également mise en évidence par les résultats des tests statistiques appliqués aux pluies annuelles dans les travaux de Kouassi et al. (2008) et Goula et al. (2006).

Le démarrage progressif de la saison des pluies est la conséquence du mouvement, en zone guinéenne et soudano-guinéenne, de la mousson humide qui s'étend du Sud-Ouest vers le Nord-Est de la Côte d'Ivoire. Ces résultats sont conformes à ceux de Noufé et al. (2015), lesquels ont montré que l'installation de la grande saison des pluies, dans l'Est ivoirien, se fait d'abord dans les latitudes inférieures avant de progresser vers les latitudes supérieures. Les travaux de Kanga (2017) ont confirmé ce constat en indiquant l'existence d'une relation directe entre le climat et la progression de la mousson qui entre dans le pays par la zone de Tabou (Sud-Ouest) et traverse le pays jusqu'à la limite frontalière Nord-Est (Zone de Bouna).

En étudiant les saisons des pluies potentiellement utiles au Togo, Adewi et al. (2010) ont abouti aux mêmes conclusions. Ces auteurs ont montré que la grande saison des pluies, démarre généralement dans la $2^{\text {ème }}$ quinzaine d'avril pour les stations situées entre $7^{\circ}$ et $9^{\circ} \mathrm{N}$, c'est-à-dire du Centre au NordEst de la dorsale togolaise. Cette zone appartient pratiquement au même espace géographique que la zone de transition ivoirienne. En outre, ce résultat est très proche des dates moyennes de début des saisons pluvieuses mises en évidence dans cette étude. Par ailleurs, lors de son étude dans le quart NordEst ivoirien, Kanga (2017) a montré que sur les stations de Bouaké, Katiola et Dabakala, la grande saison des pluies, annonçant le démarrage de la saison culturale, débute plus tôt à partir du mois de mars par rapport aux stations de Ouangolodougou et de Bouna qui sont situées plus au Nord et pour lesquelles le début de la saison est plus tardif. Ces résultats sont en accord avec ceux mis en évidence dans cette étude pour les mêmes localités. Les résultats de cette étude indiquent que la grande saison des pluies oscille, en moyenne, entre la dernière décade de mars à Bouaké (24 mars) et la première décade d'avril à Dabakala (10 avril). Lors d'une étude sur l'incidence des changements hydroclimatiques sur l'agriculture dans les paysanneries de l'Est ivoirien, Noufé et al. (2015) ont abouti au même constat, dans la localité de Dimbokro, située au Centre-Est de la Côte d'Ivoire. Dans cette localité, la tendance 
moyenne de démarrage de la grande saison des pluies potentiellement utiles est fixée autour du 22 mars. Plus récemment, à la faveur d'une étude similaire portant sur l'impact de la variabilité pluviométrique sur la saison culturale dans la zone cotonnière ivoirienne, Dékoula et al. (2018) ont également abouti aux mêmes conclusions en indiquant qu'actuellement le démarrage moyen de la grande saison des pluies utiles se situe dans la dernière décade de mars dans la zone de transition comme l'analyse statistique l'a prouvé pour Béoumi (27 mars). Les résultats de cette étude ont également montré que la petite saison pluvieuse débute en moyenne dans le mois d'août dans toutes les localités (du 06 au 22 août). Les travaux de Noufé et al. (2015), Kanga (2017) et Dékoula et al. (2018) en Côte d'Ivoire puis ceux d'Adewi et al. (2010), ont confirmé cette tendance.

S'il est vrai que le démarrage des saisons pluvieuses s'effectue selon le gradient (Sud-Ouest-Nord-Est), pour les fins des saisons des pluies, en revanche, l'on note qu'elles interviennent précocement à Bouaké et à Dabakala et tardivement à Béoumi et à Katiola. On remarque que l'évolution des fins de saisons pluvieuses est plus aléatoire. En effet, elle ne suit pas le même gradient que celui des débuts de saisons de pluie dans la mesure où elle varie d'une zone à une autre (Dékoula et al., 2018).

Il résulte de ce qui précède que les durées des saisons pluvieuses et des cumuls pluviométriques saisonniers dans les localités étudiées sont tout aussi variables. Ces résultats démontrent que les perturbations des calendriers culturaux indiquées par Diomandé (2013) dans la zone de contact forêt-savane sont bien réelles. Cette variation spatio-temporelle de la durée et du cumul pluviométrique des saisons des pluies dans la région a été également mise en évidence par les travaux de Brou et al. (2005), Kouassi (2007) et Kouassi et al. (2018).

Les résultats d'analyse statistique descriptive, indiquent que, la grande saison pluvieuse s'installe, en moyenne, entre le 24 mars et le 07 juillet (soit 106 jours). À Béoumi, la grande saison des pluies débute, en moyenne, le $1^{\mathrm{er}}$ avril et s'achève le 11 juillet (soit 101 jours). À Katiola, la grande saison des pluies démarre, en moyenne, le 02 avril et s'achève le 08 juillet (soit 97 jours). À Dabakala, en revanche, la grande saison pluvieuse commence, en moyenne le 10 avril et se termine le 06 juillet (soit 87 jours).

À Bouaké, la petite saison pluvieuse débute en moyenne le 06 août et s'achève en moyenne le 12 octobre (soit 66 jours). À Béoumi, la petite saison s'installe, en moyenne, entre le 17 août et le 02 novembre (soit 76 jours). À Katiola, la plus petite des saisons pluvieuses démarre, en moyenne, le 22 août et prend fin le 17 octobre (soit 56 jours). Quant à Dabakala, la saison commence, en moyenne, le 20 août et se termine le 21 octobre (soit 62 jours). Ces résultats, notamment ceux de Bouaké et de Dabakala, sont très proches de la durée de la petite saison des pluies, enregistrée dans la commune de Savalou 
au Bénin. En effet, lors d'une étude visant à déterminer les incidences de la durée de la deuxième saison agricole sur la production de l'arachide au Bénin, Yabi et al. (2012) ont indiqué que la durée moyenne de la seconde saison pluvieuse à Savalou est de 60 jours (du 24 août au 24 octobre).

D’une manière générale, l'on observe que les saisons pluvieuses des localités de Bouaké et Béoumi sont plus longues que celles des localités septentrionales (Katiola et Dabakala) de la zone d'étude. Ces résultats obtenus, sont en phase avec ceux de Kouassi et al. (2010) et de Yao et al. (2013).

Cependant, s'il est vrai qu'en agronomie le régime pluviométrique est un facteur déterminant dans la planification des activités agricoles, d'autres paramètres non moins importants tels que la fréquence des jours pluvieux et la répartition des pluies au cours de la saison sont aussi susceptibles d'influencer les activités agricoles.

L'analyse a révélé qu'il existe dans la zone d'étude des disparités tant au niveau du nombre de jours de pluie que des jours secs, selon que l'on passe d'une localité à une autre. Ainsi, le nombre de jours pluvieux, pour les deux saisons des pluies, est plus élevé à Bouaké et à Béoumi et décroit en allant à Katiola et Dabakala. Ces résultats sont corroborés par ceux de Kouassi et al. (2010), Yao et al. (2013), Kanga (2017) et Dékoula et al. (2018), qui ont montré qu'en Côte d'Ivoire l'axe dominant des vents entraîne une décroissance des pluies du Sud-Ouest vers le Nord-Est. Il découle de ce phénomène que les séquences sèches dans chaque saison des pluies sont plus importantes à Katiola et Dabakala par rapport à Bouaké et Béoumi.

\section{Conclusion}

Cette étude a permis d'évaluer, à partir d'une approche fréquentielle, l'impact de la récession pluviométrique sur les facteurs agroclimatiques fondamentaux de la saison culturale en zone de contact forêt-savane de Côte d'Ivoire. L'analyse des données pluviométriques recueillies dans la région, sur la période 1961-2000, révèle que les moyennes des paramètres fondamentaux des deux saisons pluvieuses observées pour chacune des quatre stations présentent des différences plus ou moins significatives. Les saisons de pluies débutent plus tardivement dans les localités septentrionales du domaine d'étude (Katiola et Dabakala) et s'y achèvent plus tôt. Les longueurs des saisons, les nombres de jours pluvieux et les cumuls pluviométriques saisonniers apparaissent alors beaucoup plus élevés dans les localités situées dans le Sud de la zone d'étude (Bouaké et Béoumi), ce qui justifie le caractère moins marqué des épisodes secs saisonniers dans ces localités par rapport à celles de Katiola et Dabakala. Cette situation atteste bien que les perturbations pluviométriques saisonnières influencent différemment les pratiques paysannes et les rendements des spéculations dans la région Centre du pays. 


\section{Remerciements}

Nous tenons à remercier le CNRA (Centre National de Recherche Agronomique) pour le financement qui nous a été octroyé par le biais du programme Gestion Durable des Sols et Maîtrise de l'Eau (GDSME). Nous tenons aussi à remercier tout particulièrement le Service Météorologique du Centre National de Recherche Agronomique pour le cadre de discussions et de partage de données qu'il offre. Nous remercions également la Société d'Exploitation et de Développement Aéronautique, Aéroportuaire et Météorologique (SODEXAM) pour leur collaboration. Nous voulons remercier enfin, toutes les personnes physiques et morales qui ont contribué à l'élaboration de ce travail.

\section{References:}

1. Brou, Y. T., Akindes, F., \& Bigot, S. (2005). La variabilité climatique en Côte d'Ivoire: entre perceptions sociales et réponses agricoles. Cahiers Agricultures, 14 (6) : 533-540.

2. Brou, Y. T., Servat, E., \& Paturel, J.-E. (1998). Activités humaines et variabilité climatique: cas du sud forestier ivoirien, IAHS Publ, (252) :365-373.

3. Dékoula, S. C., Kouamé, B., N'goran, K.E., Ehounou, J.N., Yao, G. F., Kassin, E.K., Kouakou, B. J., N'guessan, B.E.J, \& Soro, N. (2018). Variabilité des descripteurs pluviométriques intrasaisonniers à impact agricole dans le bassin cotonnier de Côte d'Ivoire : cas des zones de Boundiali, Korhogo et Ouangolodougou. Journal of Applied Biosciences (130) : 13199 - 13212.

4. Dékoula, S. C., Kouamé, B., N'goran, K.E., Yao, G. F., Ehounou, J.N., \& Soro, N. (2018). Impact de la variabilité pluviométrique sur la saison culturale dans la zone de production cotonnière en Côte d'Ivoire. European scientific journal, 14(12) : 143-159.

5. Diomandé, M. (2013). Impact du changement de pluviosité sur les systèmes de production en zone de contact forêt-savane de Côte d'Ivoire. Thèse de Doctorat d'Etat, Université Félix HouphouëtBoigny d'Abidjan, 162p.

6. Dolidon, H. (2007). «La multiplicité des échelles dans l'analyse d'un phénomène d'interface nature/société. L'exemple des feux de brousse en Afrique de l'ouest ", Cybergeo : European Journal of Geography [En ligne], Environnement, Nature, Paysage, document 363, mis en ligne le 08 mars 2007, consulté le 20 novembre 2018. URL : http://journals.openedition.org/cybergeo/4805 ; DOI : 10.4000/cybergeo.4805.

7. Goula, B.T.A., Savané, I., Konan, B., Fadika, V., \& Kouadio, G.B. (2006). «Impact de la variabilité climatique sur les ressources 
hydriques des bassins de N'zo et N'zi en Côte d'Ivoire (Afrique tropicale humide) », Vertigo, vol. 1, 1-12.

8. Kanga, K. H. M. (2017). Sècheresse et vulnérabilité socio-spatiale dans le quart nord-est de la Côte d'Ivoire. Thèse unique de doctorat, Université Alassane Ouattara, Bouaké, 347p.

9. Kouamé, B. (1992). Adéquation de différents modèles globaux pluiedébit pour déterminer les apports en eau dans les zones de transition et de forêt de la Côte d'ivoire : Essai de régionalisation des paramètres. Thèse de Doctorat, $200 \mathrm{p}$.

10. Kouassi, A.M. (2007). Caractérisation d'une modification éventuelle de la relation pluie-débit et ses impacts sur les ressources en eau en Afrique de l'Ouest : cas du bassin versant du N'zi (Bandama) en Côte d'Ivoire, Thèse de Doctorat, Université de Cocody-Abidjan, Côte d'Ivoire, $210 \mathrm{p}$.

11. Kouassi, A.M., Kouamé, K.F., Goula, B.T.A., Lasm, T., Paturel, J.E., \& Biémi, J. (2008). «Influence de la variabilité climatique et de la modification de l'occupation du sol sur la relation pluie-débit à partir d'une modélisation globale du bassin versant du N'zi (Bandama) en Côte d' Ivoire », Revue Ivoirienne des Sciences et Technologie, vol. 11, 207-229.

12. Kouassi, A.M., Kouassi, N.J., Dje, K.B., Kouame, K.F., \& Biemi, J. (2018). Analyse de la durée de la saison pluvieuse en fonction de la date de démarrage des pluies en Afrique de l'Ouest : cas du bassin versant du Bandama en Côte d'Ivoire. Agronomie Africaine 30 (2) : 147-156.

13. Kouassi, A. M., Kouamé, K. F., Koffi, Y. B., Djé, K. B., Paturel, J. E., \& Oularé, S. (2010). Analyse de la variabilité climatique et de ses influences sur les régimes pluviométriques saisonniers en Afrique de l'Ouest : cas du bassin versant du N'zi (Bandama) en Côte d'Ivoire Cybergeo. European Journal of Geography, Environnement, Nature, Paysage, mis en ligne le 7 décembre 2010, modifié le 10 décembre 2010. URL: http://cybergeo.revues.org/23388. Consulté le 9 mars 2016.

14. Noufé, D., Lidon, B., Mahé, G., Servat, E., Brou, Y.T., Koli Bi, Z., \& Chaléard, J.-L. (2015). Variabilité climatique et production de maïs en culture pluviale dans l'Est ivoirien. Hydrol., Sci., J. 56 (1), pp. 152167.

15. Paturel, J.E., Servat, E., Kouamé, B., Lubès, H., Ouédraogo, M., \& Masson, J.M. (1997). Climatic variability in humid Africa along the Gulf of Guinea. Part II: an integrated regional approach. Hydrol. Sci. J., (191):16-36. 
16. Servat, E., Paturel, J. E., Lubès, H., Kouamé, B., Ouédraogo, M., Masson, J. M. (1997). Climatic variability in humid Africa along the Gulf of Guinea. Part I: detailed analysis of the phenomenon in Côte d'Ivoire, Hydrol. Sci. J., (191) : 1-15.

17. Soro, G. E., Anouman, D. G. L., BI, T. G., Srohorou, B., \& Savane, I. (2014). Caractérisation des séquences de sècheresse météorologique à diverses échelles de temps en climat de type soudanais: cas de l'extrême nord-ouest de la Cote d'Ivoire. LARHYSS Journal (18), 1112-3680.

18. Tourquebiau, E. (2015). Changement climatique et agricultures du monde. Editions CIRAD, Paris, France, 324p.

19. Yabi, I., Bio Bigou, L. B., Assogba, C., \& N'bessa, B. (2012). Production de l'arachide dans la Commune de Savalou : état des lieux et perspectives. In : Annales de la Faculté des Lettres, Arts et Sciences Humaines, Université d'Abomey-Calavi (Bénin), vol 2, n¹8, pp. 6174.

20. Yao, N. R., Oulé, A. F., \& N'goran, K.D. (2013). Etude de Vulnérabilité du Secteur Agricole face aux Changements Climatiques en Côte d'Ivoire. Rapport Final Ministère de l'Environnement et du Développement Durable de Côte d'Ivoire/PNUD : 105 p. 